\title{
IMMUNOLOCALIZATION OF BASIC FIBROBLAST GROWTH FACTOR RECEPTORS IN INTERNAL THORACIC ARTERY AND SAPHENOUS VEIN GRAFTS
}

\author{
Kyriakos St. Rammos, MD, and George J. Koullias, MD, Thessaloniki, Greece
}

Basic fibroblast growth factor (bFGF), a 146-amino acid peptide with a strong affinity for heparin, has been found to induce mitogenic responses of endothelial ${ }^{1}$ and vascular smooth muscle cells, augmenting their migratory biochemical phenotypes, ${ }^{2}$ and inducing capillary blood vessel growth. ${ }^{3}$ Because of these mitogenic, migrating, and angiogenic properties, bFGF has ben inferred by several investigators to play a central role in pathobiochemical models of intimal hyperplasia and consequent vascular remodeling. ${ }^{2,4}$ The biologic actions of $b F G F$ are exerted through its interaction with two different $\mathrm{bFGF}$ receptor (bFGFR) classes, which are located either on the target-

From the Department of Cariothoracic Surgery, Aristotle University of Thessaloniki, Medical School, AHEPA General Hospital, Thessaloniki, Greece.

Received for publication Nov. 18, 1996; accepted for publication Nov. 27, 1996.

Address for reprints: Kyriakos St. Rammos, MD, 101 Mitropoleos St., Thessaloniki 546 22, Greece.

J Thorac Cardiovasc Surg 1997;113:1111-2

Copyright (C) 1997 by Mosby-Year Book, Inc.

$0022-5223 / 97 \$ 5.00+0 \quad \mathbf{1 2 / 5 4 / 7 9 6 0 8}$ cell membrane (high-affinity bFGFRs) or in the extracellular matrix (low-affinity bFGFRs).

The superiority in long-term patency of internal thoracic artery (ITA) grafts versus saphenous vein (SV) grafts is in part attributable to the advantageous histologic and biologic characteristics of the former. These characteristics conduce to the absence of significant intimal hyperplasia and atherosclerotic changes. Because of the previously mentioned role of bFGF in vascular remodeling, we studied immunohistochemically the expression bFGFRs in ITA and SV tissue samples obtained from patients during coronary artery bypass grafting.

Methods. Seventy-six patients undergoing coronary artery bypass grafting with both ITA and SV conduits were recruited for this study. After median sternotomy, graft preparation, and graft harvesting, two $3 \mathrm{~mm}$ long vascular portions (one arterial and one venous) were obtained from the distal parts of the two grafts for each patient, placed immediately in neutral $10 \%$ formalin, and subsequently embedded in paraffin. Two $5 \mu \mathrm{m}$ thick tissue sections for each patient (one ITA and one SV) were subjected to a three-stage avidin-biotin immunoperoxidase staining technique with the use of an anti-bFGFR monoclonal antibody that selectively binds to the high-

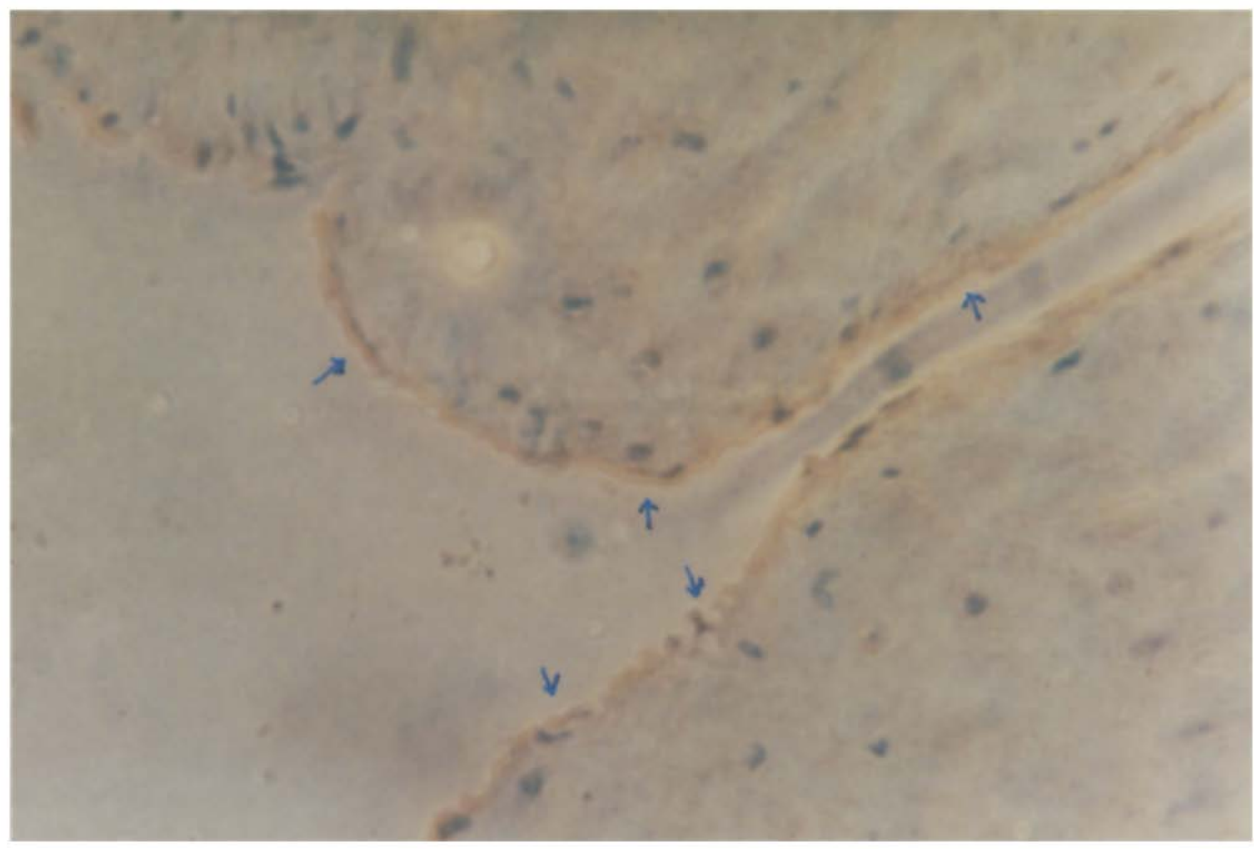

Fig. 1. Strong endothelial SV graft bFGFR immunostaining. Arrows indicate brownish immunostaining (original magnification $\times 140$ ). 


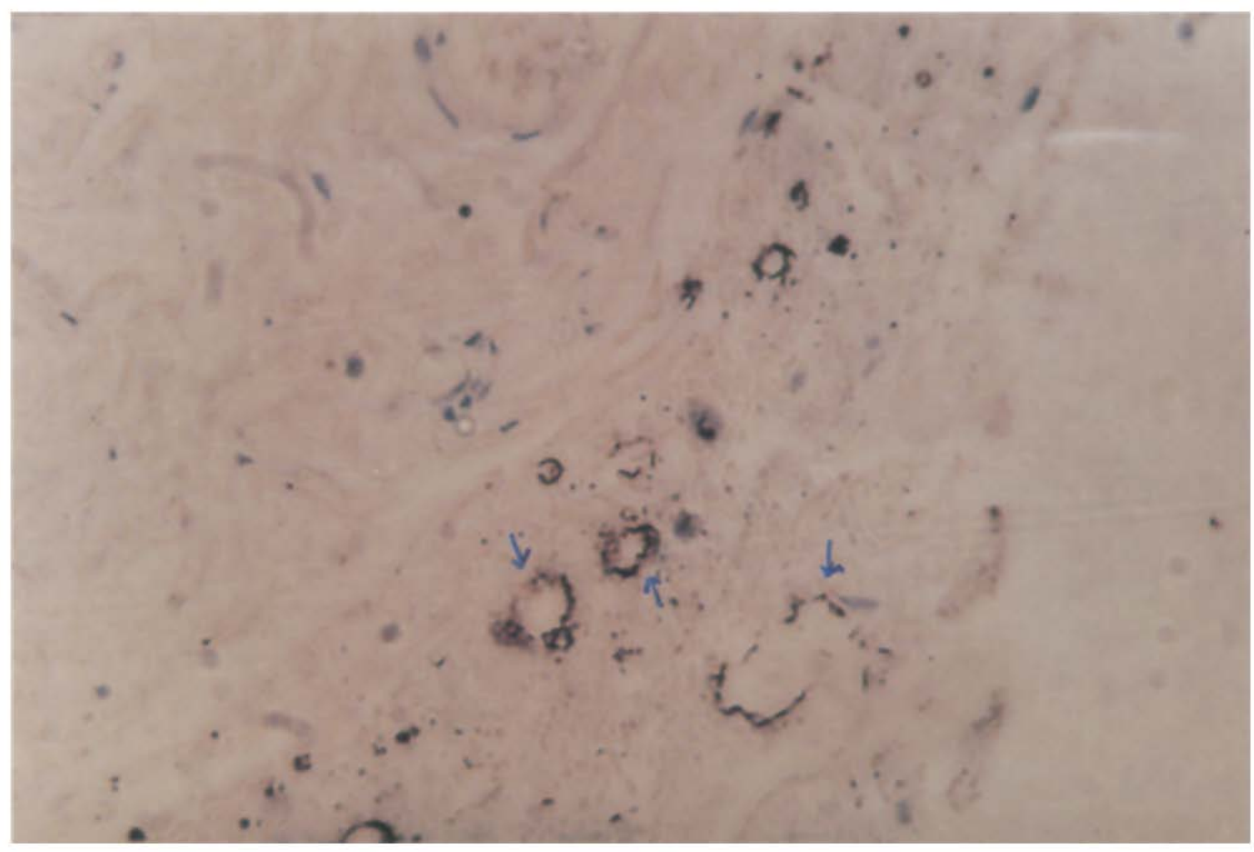

Fig. 2. Intense vasa vasorum bFGFR presence in the periadventitial connective tissue of ITA graft. Arrows indicate brownish immunostaining (original magnification $\times 200$ ).

affinity bFGFRs (Ab-1; Oncogene Science, Inc., Uniondale, N.Y.). Negative control sections were prepared by replacing the primary antibody with normal swine serum, and sections from previously known positive prostatic carcinoma tissue were used as positive controls.

The derived results were then assessed with regard to the presence of immunostaining in the three layers of the vascular wall and the adjacent periadventitial connective tissue. The $\chi^{2}$ test was used to define possible significant differences regarding the localization of immunostaining in the intima, media, or externa. A $p$ value equal to or less than 0.05 was considered statistically significant.

Results. In the endothelium, bFGFRs were found in 29 SV grafts $(40.84 \%)$ and in eight ITA grafts $(10.8 \% ; p<$ 0.05; Fig. 1). In the media, bFGFR immunoreactivity was detected in $47(66.19 \%)$ of the SVs and was absent in the vascular smooth-muscle cells of the ITA $(p<0.05)$. In the adventitia, $61(85.91 \%)$ of the SV grafts and $48(64.86 \%)$ of the ITA grafts had positive staining for bFGFR. When sole focus was placed on the small periadventitial vessels, however, the bFGFR immunoreactivity was found to be located exclusively in the ITA grafts (36 ITA grafts [48.64\%] vs one SV graft [1.38\%]; Fig. 2).

Discussion. Until now, histologic differences, such as thicker media and an incomplete internal elastic lamina, and biochemical differences, including increased thromboxane $\mathrm{A}_{2}$ and decreased prostacyclin and nitric oxide production, were the chief explanations given for the clinically established inferiority in long-term patency rates of SV versus ITA grafts. The results of this study indicate, at an immunohistochemical level of accuracy, that ITA and SV have an important molecular biologic difference. SV has a significantly greater presence of bFGF receptors in the endothelium and in the muscularis than does ITA.
This difference between SV and ITA was also noted in a recent animal study by Nguyen and colleagues. ${ }^{5}$ They stated that because increased levels of bFGF in the vessel wall of injured vessels were shown to accelerate the intimal hyperplasia process, the increased number of bFGF receptors in SV (found both in their study and in our own) might trigger the same process. These increased bFGFR levels in the SV wall could explain the inferiority of long-term results of SV as a coronary bypass conduit.

Further molecular studies are required to elucidate the role of bFGF in intimal hyperplasia. We hope that insight into the mechanism described here will, in the near future, provide clinicians with molecular tools to help prevent or delay the process of SV graft failure.

\section{REFERENCES}

1. Schweigerer L, Neufeld G, Freiman J, Abraham JA, Fiddes JC, Gospodarowicz D. Capillary endothelial cells express basic fibroblast growth factor, a mitogen that promotes their own growth. Nature 1987;325:257-9.

2. Klagsbrun M, Edelman ER. Biological and biochemical properties of fibroblast growth factors: implications for the pathogenesis of atherosclerosis. Arteriosclerosis 1989;9:269-78.

3. Folkman J, Klagsbrun M. Angiogenic factors. Science 1987; 235:442-7.

4. Lindner V, Lappi DA, Baird A, Majack RA, Reidy MA. Role of basic fibroblast growth factor in vascular lesion formation. Cir Res 1991;68:106-13.

5. Nguyen HC, Grossi EA, LeBoutillier M III, Steinberg BM, Rifkin DB, Baumann FG, et al. Mammary artery versus saphenous vein grafts: assessment of basic fibroblast growth factor receptors. Ann Thorac Surg 1994;58:308-11. 